\title{
Comparison of ground reaction forces and joint kinematics between three different tempos during push-up exercise
}

\author{
Yahya Berk Degirmen ${ }^{1}$, Berkant Erman ${ }^{1}$, Mehmet Zeki Ozkol ${ }^{2}$, Faik Vural ${ }^{2}$, Tolga Aksit ${ }^{2}$ \\ 1 Department of Sport Health Sciences, Institute of Health Sciences, Ege University, 35050 Bornova, Izmir, Turkey; \\ 2 Coaching Education Department, Faculty of Sport Sciences, Ege University, 35050 Bornova, Izmir, Turkey
}

\begin{abstract}
Study aim: This study was aimed to analysis in detail how different tempos [2:0:2 (30 bpm), 1:0:1 (60 bpm), Explosive (EXP)] effect to ground reaction forces (vGRF) and joint kinematics of push-up exercise (PUP).

Material and methods: Twenty-four recreationally male athletes (age: $24.9 \pm 3.6$ years) participated in this study. Kinetic and kinematic data were obtained by load-cells and a motion analysis software. Data was analysed from a single repetition which is showed peak vGRF of dominant side during PUP. Joint velocities were calculated by taking the difference between the descent and ascent phases.

Results: There was significant difference between 2:0:2 (30 bpm) - EXP in terms of dominant side of shoulder ( $\mathrm{p} \leq 0.02)$ and between 1:0:1 (60 bpm) - EXP in the dominant elbow joint displacements $(\mathrm{p} \leq 0.05)$. The velocity differences between the descent and ascent phases of shoulder and elbow joints were found statistically significant between tempos ( $\mathrm{p} \leq 0.05)$. In terms of range of motion $(\mathrm{ROM})$ of right and left side, there was significant differences between tempos $(\mathrm{p} \leq 0.001)$. No significant differences were found between all tempos in the ascent phase of right-left and left descent phase in terms of average vGRF $(\mathrm{p}>0.05)$ except right descent average vGRF $(\mathrm{p} \leq 0.02)$.

Conclusions: In conclusion, right-left sides of ROM was used most effectively in 2:0:2 (30 bpm) and 1:0:1 (60 bpm) tempos. Less displacement was also observed in EXP and when tempo increased percentage of peak vGRF (at elbow flexion phase for right-left sides) to total repetition decreased. Highest ascent and descent phase velocity differences (for right-left sides) and highest peak vGRF (elbow flexion phase) observed in EXP. This study shows that increasing tempo will result in more unsteady joint kinematics and more vGRF, so if the goal is controlled and safe PUP, tempo should be slow.
\end{abstract}

\section{Keywords: Biomechanics - Body weight exercise - Motion analysis - Explosive exercise - Performance analysis}

\section{Introduction}

Push-up exercise is one of the most popular exercises to strengthen and improve endurance the upper body. This exercise consists of two main phases: flexion of the elbows (descent) and following the extension of the elbows (ascent). In the standard push-up execution, pectoralis major, triceps brachii and anterior deltoid muscles work as primary mover, serratus anterior and upper trapezius include five or more muscles works as stabilizers and synergists. In addition to these, including the rectus abdominis and external oblique muscles are involved as core muscles [4]. There are many separately important reasons why push-up exercise is popular, and it has wide usage. Such as, easy to perform with body weight (BW) only, no need any equipment and it is possible to perform many muscles at the same time as mentioned above.

There are also many push-up exercise variations, and these variations may vary depending on the training goal or the individuals' fitness level. Some of those; performing the exercise at different velocities $[5,8,16]$, changing posture and hand positions on the ground $[2,7,10]$, performing exercise at different ground elevation [10], changing ground surface [11] or on knee push-up [5-7]. The push-up is a closedchain exercise and although the performing is simple, there are important points to be considered like any exercise.

Chou et al. [1] found that a significant increase in the posterior shearing force and extension moment of elbow at 84 beats per minute $(\mathrm{bpm})$ compared to others $(60 \mathrm{bpm}$ 
and $48 \mathrm{bpm}$ ), and this result may enhance the risk of injury via the increase in the load on the triceps brachii. In the same study, also found that lower-tempo (48 bpm) pushup can significantly reduce the load on the elbow joint and this tempo increases muscle activation on biceps brachii, triceps brachii and posterior deltoid muscle 1.62, 1.39 and 1.99 times than fast push-up tempo, respectively. In addition to these, muscle activations at slow tempo push-ups also found that triceps brachii 1.47 , biceps brachii 2.43 , anterior deltoid 1.42, pectoralis major 1.48 and posterior deltoid 1.91 times more active than the fast tempo pushups and also the slow tempo delayed the onset of fatigue [8]. Yoo et al. [15] found that push-up exercise performed at different tempo also caused a change in the activation of the serratus anterior muscle, showing that the fast tempo (2s for each push-up) had less activation than the slower tempo (3s for each push-up). Gouvali and Boudolos [7] also showed that placing the hands shoulder width and $30 \%$ more of the forearm length posteriorly was more active for the pectoralis major and placing the hands at $50 \%$ adduction of shoulder's width showed that the triceps was more active than the normal position. In the same study, highest maximum vertical force and average vertical force was found statistically significant and higher than the other five versions of push-up $297.3 \pm 92 \mathrm{~N}$ and $133.2 \pm 42.2 \mathrm{~N}$ in the normal position, respectively. Previous studies also showed that relative load of push-up $69 \%$ of BW in top, $75 \%$ of BW in bottom and $49 \%$ of BW in knees bent position [4,5]. Wang et al. [13] found the peak ground reaction forces (GRF) (from 120\% to $121 \% \mathrm{BW}$ ) in the ballistic push-ups. In the push-up exercise, a significant increase was also observed in the triceps and pectoralis major muscle thickness after the 8-week program [9]. In a study on general health related to push-ups in active adult men, it was observed that individuals with forty or more push-ups capacity had $96 \%$ reduction in relation to cardiovascular disease compared to an individual who could perform ten or less push-ups (IRR: 0.04; 95\%CI: 0.01-0.36) [14]. There was also 1.34 and 1.33 times more repetitions were obtained in the high (7 push-up/10 s) and regular (5 pushup/10 s; 1:0:1) tempo rather than slow tempo (4 pushup/10 s) [1]. Considering these studies, it may be concluded that slow push-up tempo increases muscle activation more. However, during these evaluations, range of motion (ROM) and joint velocity issues were not emphasized at the same time. In this sense, observing the change of these parameters may also contribute to practitioners.

In general, a detailed examination of the kinematics of the right-left shoulder and elbow joints was not well documented. However, such as joint loads, GRF and electromyogram activations in different positions (posture etc.) with usually the dominant side was analysed. In the present study, how the joint velocities changed in the ascent and descent phase and how the vertical GRF (vGRF) and the ROM of dominant and non-dominant side showed differentiation in three (1:0:1, 2:0:2, Explosive) tempos were investigated. Therefore, by observing these changes, practitioners may perform the push-up exercise more efficiently. It is hypothesised that i) the displacement of the joints would be different at all tempos, ii) ROM would be less at the fastest tempo rather than at other tempos, iii) the difference between the joint velocities in the descent and ascent phases would be observed greatest when tempo increases, iv) vGRF of performance would be higher when tempo increases. Understanding these situations will inform practitioners about the situations that they should pay attention to when planning their exercises.

\section{Material and methods}

\section{Participants}

The present investigation was designed as a cross-sectional, within subject study. The sample size was calculated using $G^{*}$ Power 3.1.9 [power size $(1-\beta)=0.80$, the effect size $(f)=0.40$, type- 1 error $(\alpha)=0.05$, number of groups $=1$, number of measurements $=3$, cor. among rep. measure $=0.5$, correction $\varepsilon=1$ ], depends on calculation of sample size was found twenty-eight. However, twenty-four recreationally male athletes (Ages $-24.9 \pm 3.6$ years, Body height $-178 \pm 6.9 \mathrm{~cm}$, Body mass $-80.5 \pm 10.5 \mathrm{~kg}$, Upper arm fat free mass $-8.89 \pm 1.05 \mathrm{~kg}$, Trunk fat free mass $-37.14 \pm 3.79 \mathrm{~kg}$ ) volunteered and gave their informed consent to take part in the study. Therefore, post-hoc analysis was carried out and power of present study was found 0.74. Anthropometric data obtained from a body composition analysis device (Tanita BC 418, Tanita Corp., Tokyo, Japan) which is using the bioelectric impedance method. All participants with a history of resistance exercise for at least one year and each participant had a right dominant hand. The inclusion criteria for this study were defined as (a) had no chronic limb discomfort or limitations that had a negative effect on push-up kinematics (e.g., lateral epicondylitis etc.) (b) any serious injury or had not undergone surgery in the past year (c) performing push-up exercises at least two times per week. The experimental procedures undertaken were approved by Ege University's Medical Research Ethics Committee (protocol code 16-3.1/9) and are in agreement with the principles of the Declaration of Helsinki. The participants were briefed on the study procedures and informed consent was obtained before they took part in the study.

\section{Procedures}

Push-up exercise was performed using three different tempos which were 2:0:2, 1:0:1 and explosive in three different sessions at 48-h intervals to avoid fatigue effects on upper limb. Since the participants got used to the push- 
up movement, the time between sessions was chosen as 48 hours. No participants reported delayed-onset muscle soreness during sessions. In the first session, cadence was 30 beats per minute (bpm) (2:0:2) which signifies a twocount eccentric motion followed immediately by a twocount concentric motion, without any count for rest before beginning the next repetition and this method was used for 1:0:1 in second session. There was no given tempo for explosive exercise (EXP) in third session and participants were asked to perform the exercise as maximum effort as possible. All participants performed each session in the morning between 10:00-12:00 AM. The duration to complete of the sessions was approximately $45 \mathrm{~min}$ for each participant. In the first session (2:0:2, $30 \mathrm{bpm})$, after the participants had done 10 min self-selected dynamic warm up, retroreflective markers were positioned on acromion (shoulder), lateral epicondyle (elbow) and ulnar styloid (wrist) before each session and they applied enough repetitions to adapt the tempo (except explosive tempo). After the participants took their positions on the load-cells (hands) and elevations (feet) for push-up, the digital metronome (G. Master AMT-560, China) was started. The push-up exercises performed at prone position, open elbow position (for camera recording angular kinematics), hands shoulder-width apart and knees were not bent. The researchers briefed about need to use as much full range of motion (ROM) as during each session and paid attention to this during sessions. If limitations in the usage of ROM were noticed by the researchers, verbal courage was given to the participant. In addition, hip angle was not checked with a camera during sessions. However, participants were proficient in keeping the hip stable. Moreover, the upward or downward movement of the hip was controlled by the front camera. This situation can be considered as one of the limitations of the study. Same procedures were also applied for the second (1:0:1, $60 \mathrm{bpm})$ and third sessions (explosive tempo). To obtain vertical ground reaction forces (vGRF, right and left sides), two load-cells (SPS Platform Type $60 \times 60 \mathrm{~cm}$, CAS Electronic Industry and Trade Inc., Korea) were used. Load-cells were connected to a data acquisition system (BIOPAC MP150, Biopac Systems, Goleta, CA, USA) and the system was reset to zero (load cell signal range; $0-0.15 \mathrm{~kg}$ ) before the participant put their hands on the load-cells. For obtaining to same height ground with load-cells, two elevations were used. In order to ensure that each load cell and elevation were placed symmetrically next to each other, height was carefully adjusted using a spirit level. The push-up exercise was captured by a camera (Philips SPC620NC, Amsterdam, Netherlands) with a frequency of $30 \mathrm{~Hz}$. Since data acquisition system (AcqKnowledge 4.2 Software and BIOPAC MP150) does not work synchronously with the higher resolution camera, the video had to recorded with $30 \mathrm{~Hz}$ due to this issue. The camera placed at height of

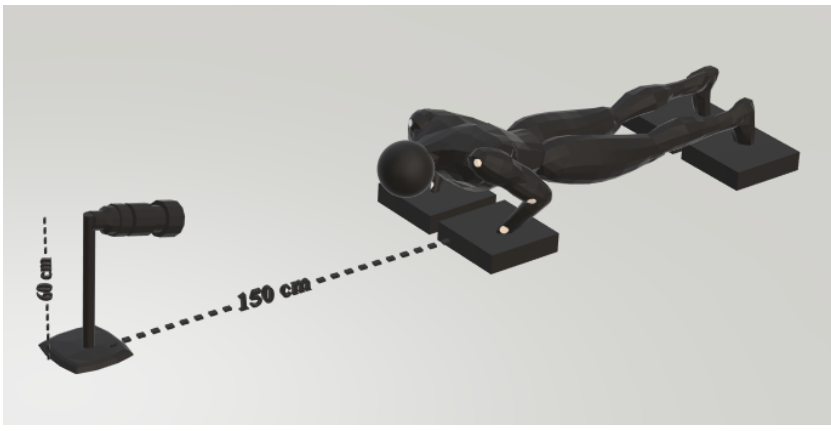

Figure 1. Illustration of observation of push-up exercise

$60 \mathrm{~cm}$ and distance of $150 \mathrm{~cm}$ in front of the participants with open-elbow position (about $\sim 90^{\circ}$ from camera), so that the retroreflective markers could be monitored easily (Figure 1).

The vGRF was recorded continuously at $2000 \mathrm{~Hz}$ with using AcqKnowledge (4.2) software. Data filtered using a low pass infinite impulse response (IIR) filter at $50 \mathrm{~Hz}$. The first three and the last two repetitions were excluded to determining the appropriate push-up for adjust the exercise tempo and fatigue effect. Thus, false vGRF data was eliminated and the proper single repetition were considered in the analysis. This single repetition was analysed from a repetition which is showed peak vGRF of dominant side. The vGRF variables were reported as Newton. This method was applied for each different tempo. The ROM data were analysed with Kinovea motion analysis software ${ }^{\circledR}$ (downloaded at: www.kinovea.org, accessed on 05 January 2021). The range of motion (ROM) was obtained from three points (retroreflective markers as mentioned above) and the vertical velocity of movement $\left(\mathrm{m} \cdot \mathrm{s}^{-1}\right)$, total displacement of shoulder and elbow joints $(\mathrm{cm})$, was also obtained via same software. The absolute differences in peak velocities $\left(\mathrm{m} \cdot \mathrm{s}^{-1}\right)$ during the descent and ascent phases of shoulder and elbow joints were considered for analysis. Because, as might be expected, at different tempos, the joint velocities would also be different. These differences were calculated from peak velocities of descent and ascent phases (e.g., peak velocity difference of left shoulder calculated by peak ascent velocity minus peak descent velocity).

\section{Statistical analysis}

The data were presented as the mean and standard deviation. To assess difference of kinematics between the three push-up tempos analyses of variance (one-way repeated ANOVA) was performed. Greenhouse-Geisser adjustments of the $p$-values were reported due to sphericity assumption was violated [12]. Partial eta squared $\left(\eta_{\mathrm{p}}^{2}\right.$ was used as an effect size for the present study and categorised as small (0.01-0.06), medium (0.06-0.14) and large effect $(>0.14)$ [3]. If the ANOVA result was found significant, Holm-Bonferroni adjustment were used to identify 
differences. Pearson correlation coefficients (r) were used to determine the relationship between the kinematic/kinetic measurements (ROM, peak velocity differences, displacements and peak vGRF) and lean body mass of participants (trunk and arms). Data analysis was performed using SPSS software (IBM SPSS Statistics for Windows, Version 25.0. Armonk, NY: IBM Corp). The statistical significance level was set at $\mathrm{p} \leq 0.05$.

\section{Results}

Participants performed push-up exercise $11 \pm 3.49$ repetitions at 2:0:2 $(30 \mathrm{bpm}), 21 \pm 7.48$ repetitions at 1:0:1 $(60 \mathrm{bpm})$ and $23 \pm 12.78$ repetitions at explosive tempo (EXP). In the EXP, participants performed the pushup exercise in $0.82 \pm 0.15 \mathrm{~s}(146 \mathrm{bpm})$. The results also showed that for the right side, there were vertical ground reaction force $(\mathrm{vGRF})$ differences at elbow flexion phase between 2:0:2-1:0:1, 1:0:1-EXP and 2:0:2-EXP was $32.81 \pm 6.31 \mathrm{~N}(9.51 \%), 200.54 \pm 16.83 \mathrm{~N}(52.63 \%)$ and $233.35 \pm 17.59 \mathrm{~N}(67.14 \%)$, respectively. For the left side of peak vGRF (elbow flexion phase), the differences between 2:0:2-1:0:1, 1:0:1-EXP and 2:0:2-EXP were $35.18 \pm 5.37 \mathrm{~N}(18.84 \%), 204.69 \pm 15.68 \mathrm{~N}(53.94 \%)$ and $239.88 \pm 16.23 \mathrm{~N}(69.56 \%)$, respectively. When vGRF was normalized with body weight, total vGRF of right and left sides showed $87 \%, 96 \%$ and $148 \%$ of body weight at 2:0:2, 1:0:1 and EXP, respectively. For the dominant (right) shoulder displacements, 9.24\% significant difference was observed between 1:0:1-EXP and there were $8.32 \%$ differences between 2:0:2-EXP in dominant (right) elbow displacement. The one-way repeated ANOVA results showed that there was significant difference in the total displacement of dominant side of shoulder $(\mathrm{p} \leq 0.03)$ and elbow joint $(\mathrm{p} \leq 0.02)$. When dominant (right) shoulder displacement takes into consideration, there was found a significant difference between 1:0:1-EXP of exercise $(\mathrm{p}=0.02)$, and there was only found significant difference between 2:0:2-EXP for dominant (right) elbow displacement $(p \leq 0.05)$. The repetition of the peak vGRF at elbow flexion phase was examined and a significant difference was found between 2:0:2-1:0:1 ( $p \leq 0.002)$. There were also significant differences between ascent and descent phases of performance at shoulder and elbow peak velocities $(p \leq 0.05)$. Range of motions of right $(p \leq 0.001)$ and left side $(\mathrm{p} \leq 0.001)$ showed significant differences between different exercise tempos. When the descent and ascent phases are considered separately, only in the right descent average of vGRF was showed significant difference ( $p \leq 0.02)$. Left descent, right ascent, and left ascent average of vGRFs did not show any significant differences $(p>0.05)$. There were significant differences between tempos in the normalized total peak vGRF (right-left sides) $(p \leq 0.001)$ and all comparisons found statistically significant $(\mathrm{p} \leq 0.001)$. All mean \pm standard deviations $(\mathrm{M} \pm \mathrm{SD})$, ANOVA results and multiple comparisons were also shown in Table 1.

Table 1. The one-way repeated ANOVA results and multiple comparisons

\begin{tabular}{|c|c|c|c|c|c|c|}
\hline \multirow{4}{*}{ Measurement } & \multirow{4}{*}{$F_{2,46}$} & \multirow{4}{*}{$\begin{array}{l}\text { Repeated } \\
\text { ANOVA } p\end{array}$} & \multirow{4}{*}{$\eta_{\mathrm{p}}^{2}$} & Tempo $(\mathrm{M} \pm \mathrm{SD})$ & \multirow{4}{*}{$\begin{array}{l}\text { Mean } \\
\text { Diff. }\end{array}$} & \multirow{4}{*}{$\begin{array}{c}\text { Holm- } \\
\text { Bonferron } \\
p\end{array}$} \\
\hline & & & & $2: 0: 2-1: 0: 1$ & & \\
\hline & & & & 1:0:1-EXP & & \\
\hline & & & & 2:0:2-EXP & & \\
\hline \multirow{3}{*}{ Dom. Shoulder Displacement [cm] } & \multirow{3}{*}{3.659} & \multirow{3}{*}{$0.03 *$} & \multirow{3}{*}{0.13} & $69.81 \pm 14.38-69.73 \pm 10.92$ & 0.08 & 1.0 \\
\hline & & & & $69.73 \pm 10.92-63.28 \pm 13.10$ & 6.44 & $0.02 *$ \\
\hline & & & & $69.81 \pm 14.38-63.28 \pm 13.10$ & 6.52 & 0.14 \\
\hline \multirow{3}{*}{ Dom. Elbow Displacement [cm] } & \multirow{3}{*}{4.215} & \multirow{3}{*}{$0.02 *$} & \multirow{3}{*}{0.15} & $23.78 \pm 5.77-24.11 \pm 5.63$ & 0.33 & 1.0 \\
\hline & & & & $24.11 \pm 5.63-21.8 \pm 5.77$ & 1.98 & 0.08 \\
\hline & & & & $23.78 \pm 5.63-21.8 \pm 5.77$ & 2.31 & $0.05^{*}$ \\
\hline \multirow{3}{*}{$\begin{array}{l}\text { Percentage of Peak vGRF Repetition } \\
\text { to Total Repetition }[\%]\end{array}$} & \multirow{3}{*}{4.675} & \multirow{3}{*}{$0.01 *$} & \multirow{3}{*}{0.16} & $57.89 \pm 16.21-53.66 \pm 20.04$ & 4.22 & 1.0 \\
\hline & & & & $53.66 \pm 20.04-43.62 \pm 15.65$ & 10.04 & 0.11 \\
\hline & & & & $57.89 \pm 16.21-43.62 \pm 15.65$ & 14.27 & 0.21 \\
\hline \multirow{3}{*}{ Right Shoulder Peak Velo. Diff. [m $\left.\cdot \mathrm{s}^{-1}\right]$} & \multirow{3}{*}{17.847} & \multirow{3}{*}{$<0.001^{\dagger}$} & \multirow{3}{*}{0.43} & $0.05695 \pm 0.05698-0.10 \pm 0.07$ & 0.046 & 0.09 \\
\hline & & & & $0.10 \pm 0.07-0.287 \pm 0.237$ & 0.184 & $0.02 *$ \\
\hline & & & & $0.05695 \pm 0.05698-0.287 \pm 0.237$ & 0.23 & $0.001 *$ \\
\hline \multirow{3}{*}{ Left Shoulder Peak Velo. Diff. $\left[\mathrm{m} \cdot \mathrm{s}^{-1}\right]$} & \multirow{3}{*}{14.922} & \multirow{3}{*}{$<0.001^{\dagger}$} & \multirow{3}{*}{0.39} & $0.0596 \pm 0.0608-0.101 \pm 0.093$ & 0.042 & 0.33 \\
\hline & & & & $0.101 \pm 0.093-0.276 \pm 0.23$ & 0.174 & $0.003 *$ \\
\hline & & & & $0.0596 \pm 0.0608-0.276 \pm 0.23$ & 0.217 & $0.01 *$ \\
\hline
\end{tabular}


Table 1. cont.

\begin{tabular}{|c|c|c|c|c|c|c|}
\hline \multirow{4}{*}{ Measurement } & \multirow{4}{*}{$F_{2,46}$} & \multirow{4}{*}{$\begin{array}{l}\text { Repeated } \\
\text { ANOVA } p\end{array}$} & \multirow{4}{*}{$\eta_{\mathrm{p}}^{2}$} & Tempo $(\mathrm{M} \pm \mathrm{SD})$ & \multirow{4}{*}{$\begin{array}{l}\text { Mean } \\
\text { Diff. }\end{array}$} & \multirow{4}{*}{$\begin{array}{c}\text { Holm- } \\
\text { Bonferroni } \\
p\end{array}$} \\
\hline & & & & $2: 0: 2-1: 0: 1$ & & \\
\hline & & & & 1:0:1-EXP & & \\
\hline & & & & 2:0:2-EXP & & \\
\hline \multirow{3}{*}{ Right Elbow Peak Velo. Diff. [m $\cdot \mathrm{s}^{-1}$ ] } & \multirow{3}{*}{8.176} & \multirow{3}{*}{$<0.001^{\dagger}$} & \multirow{3}{*}{0.26} & $0.029 \pm 0.02-0.060 \pm 0.038$ & 0.031 & $0.005^{*}$ \\
\hline & & & & $0.060 \pm 0.038-0.117 \pm 0.135$ & 0.057 & 0.08 \\
\hline & & & & $0.029 \pm 0.02-0.117 \pm 0.135$ & 0.088 & $0.01 *$ \\
\hline \multirow{3}{*}{ Left Elbow Peak Velo. Diff. $\left[\mathrm{m} \cdot \mathrm{s}^{-1}\right]$} & \multirow{3}{*}{6.240} & \multirow{3}{*}{$0.003^{*}$} & \multirow{3}{*}{0.21} & $0.031 \pm 0.026-0.049 \pm 0.04$ & 0.018 & 0.22 \\
\hline & & & & $0.049 \pm 0.04-0.08 \pm 0.072$ & 0.030 & 0.1 \\
\hline & & & & $0.031 \pm 0.026-0.08 \pm 0.072$ & 0.049 & $0.02 *$ \\
\hline \multirow{3}{*}{ Right ROM $\left[^{\circ}\right]$} & \multirow{3}{*}{20.400} & \multirow{3}{*}{$<0.001^{\dagger}$} & \multirow{3}{*}{0.47} & $112.92 \pm 12.69-113.47 \pm 12.35$ & 0.554 & 1.00 \\
\hline & & & & $113.47 \pm 12.35-99.67 \pm 15.09$ & 13.80 & $<0.001^{\dagger}$ \\
\hline & & & & $112.92 \pm 12.69-99.67 \pm 15.09$ & 13.24 & $<0.001^{\dagger}$ \\
\hline \multirow{3}{*}{ Left ROM $\left[^{\circ}\right]$} & \multirow{3}{*}{7.244} & \multirow{3}{*}{$0.002 *$} & \multirow{3}{*}{0.24} & $108.97 \pm 24.43-113.33 \pm 11.59$ & 4.35 & 0.94 \\
\hline & & & & $113.33 \pm 11.59-99.07 \pm 24.43$ & 14.26 & $<0.001^{\dagger}$ \\
\hline & & & & $108.97 \pm 24.43-99.07 \pm 24.43$ & 9.90 & 0.09 \\
\hline \multirow{3}{*}{ Right peak vGRF $[\mathrm{N}]$} & \multirow{3}{*}{151.279} & \multirow{3}{*}{$<0.001^{\dagger}$} & \multirow{3}{*}{0.86} & $347.42 \pm 51.13-380.23 \pm 65.35$ & 32.81 & $<0.001^{\dagger}$ \\
\hline & & & & $380.23 \pm 65.35-580.77 \pm 108.56$ & 200.54 & $<0.001^{\dagger}$ \\
\hline & & & & $347.42 \pm 51.13-580.77 \pm 108.56$ & 233.35 & $<0.001^{\dagger}$ \\
\hline \multirow{3}{*}{ Left peak vGRF $[\mathrm{N}]$} & & & & $345.17 \pm 53.10-380.36 \pm 58.24$ & 35.18 & $<0.001^{\dagger}$ \\
\hline & 187.097 & $<0.001^{\dagger}$ & 0.89 & $380.36 \pm 58.24-585.05 \pm 97.92$ & 204.69 & $<0.001^{\dagger}$ \\
\hline & & & & $345.17 \pm 53.10-585.05 \pm 97.92$ & 239.88 & $<0.001^{\dagger}$ \\
\hline & & & & $293.60 \pm 42.84-297.77 \pm 46.61$ & 4.17 & 0.82 \\
\hline Right Descent Avg. vGRF [N] & 7.313 & $0.02 *$ & 0.24 & $297.77 \pm 46.61-279.69 \pm 45.60$ & 18.08 & $0.004 *$ \\
\hline & & & & $293.60 \pm 42.84-279.69 \pm 45.60$ & 13.91 & 0.08 \\
\hline & & & & $293.29 \pm 43.94-295.9 \pm 45.24$ & 2.61 & 1.0 \\
\hline Left Descent Avg. vGRF [N] & 2.994 & 0.06 & 0.115 & $295.9 \pm 45.24-285.99 \pm 39.85$ & 9.91 & 0.13 \\
\hline & & & & $293.29 \pm 43.94-285.99 \pm 39.85$ & 7.29 & 0.45 \\
\hline & & & & $288.07 \pm 42.26-279.72 \pm 42.65$ & 8.35 & $0.02 *$ \\
\hline Right Ascent Avg. vGRF [N] & 2.841 & 0.069 & 0.11 & $279.72 \pm 42.65-284.04 \pm 43.16$ & 4.32 & 0.85 \\
\hline & & & & $288.07 \pm 42.26-284.04 \pm 43.16$ & 4.03 & 0.84 \\
\hline & & & & $284.03 \pm 42.32-281.16 \pm 42.43$ & 2.86 & 0.78 \\
\hline Left Ascent Avg. vGRF [N] & 0.628 & 0.53 & 0.02 & $281.16 \pm 42.43-285.05 \pm 41.23$ & 3.88 & 0.96 \\
\hline & & & & $284.03 \pm 42.32-285.05 \pm 41.23$ & 1.024 & 1.0 \\
\hline & & & & $0.87 \pm 0.04-0.96 \pm 0.07$ & 0.085 & $<0.001^{\dagger}$ \\
\hline Total Peak vGRF (Right + Left) & 183.413 & $<0.001^{\dagger}$ & 0.88 & $0.96 \pm 0.07-1.48 \pm 0.21$ & 0.521 & $<0.001^{\dagger}$ \\
\hline & & & & $0.87 \pm 0.04-1.48 \pm 0.21$ & 0.607 & $<0.001^{\dagger}$ \\
\hline
\end{tabular}

$* \mathrm{p} \leq 0.05 ;{ }^{\dagger} \mathrm{p} \leq 0.001 ; \eta_{\mathrm{p}}^{2}$ : partial eta squared effect size; $\mathrm{M} \pm \mathrm{SD}$ : Mean \pm Standard Deviation; EXP.: Explosive tempo; Diff.: Difference; $\mathrm{vGRF}$ Vertical ground reaction force; Dom: Dominant side (right); Velo. Diff.: Peak velocity differences between ascent and descent phases; ROM: Range of motion, Avg.: Average; BW: Body weight.

Bivariate correlations between peak vGRF, ROM, displacement, peak velocity differences and fat free mass (FFM) are presented in Table 2. Significant relationship was found between left elbow velocity differences (ascent- descent) and left arm FFM in EXP ( $\mathrm{r}=-0.418, \mathrm{p} \leq 0.05)$. There was also significant relationship between dominant side (right) peak vGRFs and right arm FFM in 2:0:2 (30 bpm) $(\mathrm{r}=0.576, \mathrm{p} \leq 0.03)$, and between dominant 
side (right) peak vGRFs and right FFM in 1:0:1 (60 bpm) $(\mathrm{r}=0.605, \mathrm{p} \leq 0.02)$. There were also found significant relationships between left side peak vGRF and left arm FFM in 2:0:2 ( $\mathrm{r}=0.585, \mathrm{p} \leq 0.03)$, and between left peak vGRF and left arm FFM in 1:0:1 $(\mathrm{r}=0.617, \mathrm{p} \leq 0.01)$. There were no other significant correlations between measurements and FFM.

There were also significant positive bivariate correlations between arms (right+left) FFM and total peak vGRF (right+left) $(0.559 \leq \mathrm{r} \leq 0.573, \mathrm{p} \leq 0.05)$ in $2: 0: 2$ and $1: 0: 1$. There was only significant relationship between trunk FFM and total peak vGRF in EXP $(r=0.441, p \leq 0.02)$. There were significant relationships between percentage of peak vGRF repetition to total repetition (\%) and arms FFM in $2: 0: 2(\mathrm{r}=0.558, \mathrm{p} \leq 0.02)$ and $1: 0: 1(\mathrm{r}=0.658, \mathrm{p} \leq 0.001)$. There was only significant relationship between trunk FFM and percentage of peak vGRF repetition to total repetition $(\%)$ in $\operatorname{EXP}(r=0.457, p \leq 0.025)$ (Table 3$)$.

Table 2. Bivariate correlations between measurements and right-left arms of fat free mass at three tempos

\begin{tabular}{|c|c|c|c|c|c|c|c|c|c|c|c|c|}
\hline & Tempo & & $\begin{array}{c}\text { R Peak } \\
\text { vGRF }\end{array}$ & $\begin{array}{l}\text { ROM } \\
\text { Right }\end{array}$ & $\begin{array}{c}\text { R } \\
\text { Shoulder } \\
\text { Disp. }\end{array}$ & $\begin{array}{c}\mathrm{R} \\
\text { Elbow } \\
\text { Disp. }\end{array}$ & $\begin{array}{c}\text { RS } \\
\text { Velo. } \\
\text { Diff. }\end{array}$ & $\begin{array}{l}\text { RE } \\
\text { Velo. } \\
\text { Diff. }\end{array}$ & $\begin{array}{l}\text { L Peak } \\
\text { vGRF }\end{array}$ & $\begin{array}{c}\text { ROM } \\
\text { Left }\end{array}$ & $\begin{array}{l}\text { LS Velo. } \\
\text { Diff. }\end{array}$ & $\begin{array}{c}\text { LE Velo. } \\
\text { Diff. }\end{array}$ \\
\hline \multirow{6}{*}{$\begin{array}{l}\text { Right Arm } \\
\text { FFM }\end{array}$} & \multirow{2}{*}{$2: 0: 2$} & $\mathrm{r}$ & $0.576^{*}$ & \multirow{6}{*}{$\begin{array}{c}0.182 \\
0.394 \\
-0.042 \\
0.84 \\
-0.273 \\
0.19\end{array}$} & 0.206 & \multirow{6}{*}{$\begin{array}{c}-0.36 \\
0.869 \\
-0.118 \\
0.581 \\
-0.193 \\
0.367\end{array}$} & 0.216 & 0.203 & & & & \\
\hline & & $\mathrm{p}$ & 0.003 & & 0.335 & & 0.311 & 0.341 & & & & \\
\hline & \multirow{2}{*}{$1: 0: 1$} & $\mathrm{r}$ & $0.605^{*}$ & & -0.108 & & -0.073 & -0.383 & & & & \\
\hline & & $\mathrm{p}$ & 0.002 & & 0.615 & & 0.736 & 0.065 & & & & \\
\hline & \multirow{2}{*}{ EXP } & $\mathrm{r}$ & 0.344 & & 0.145 & & 0.240 & -0.04 & & & & \\
\hline & & $\mathrm{p}$ & 0.10 & & 0.500 & & 0.259 & 0.986 & & & & \\
\hline \multirow{6}{*}{$\begin{array}{l}\text { Left Arm } \\
\text { FFM }\end{array}$} & \multirow{2}{*}{$2: 0: 2$} & $\mathrm{r}$ & & & & & & & $0.585^{*}$ & -0.107 & 0.077 & -0.016 \\
\hline & & $\mathrm{p}$ & & & & & & & 0.003 & 0.618 & 0.720 & 0.940 \\
\hline & \multirow{2}{*}{ 1:0:1 } & $\mathrm{r}$ & & & & & & & $0.617^{*}$ & -0.317 & -0.039 & 0.037 \\
\hline & & $\mathrm{p}$ & & & & & & & 0.001 & 0.132 & 0.855 & 0.863 \\
\hline & \multirow{2}{*}{ EXP } & $\mathrm{r}$ & & & & & & & 0.256 & -0.292 & 0.236 & $-0.418^{*}$ \\
\hline & & $\mathrm{p}$ & & & & & & & 0.228 & 0.166 & 0.267 & 0.042 \\
\hline
\end{tabular}

* $\mathrm{p} \leq$ 0.05; EXP.: Explosive tempo; FFM: Fat free mass; Disp.: Displacement; R: Right; L: Left; vGRF: Vertical ground reaction force; ROM: Range of motion; RS: Right shoulder; LS: Left shoulder; RE: Right elbow; LE: Left elbow; Velo. Diff.: Peak velocity differences between ascent and descent phases.

Table 3. Bivariate correlations between measurements and arms and trunk of fat free mass at three tempos

\begin{tabular}{|c|c|c|c|c|}
\hline & Tempo & & $\mathrm{R}+\mathrm{L}$ Total Peak vGRF & $\begin{array}{c}\text { Percentage of Peak vGRF Repetition } \\
\text { to Total Repetition [\%] }\end{array}$ \\
\hline \multirow{6}{*}{ Arms' $(\mathrm{R}+\mathrm{L}) \mathrm{FFM}$} & \multirow{2}{*}{$2: 0: 2$} & $\mathrm{r}$ & $0.559 *$ & $0.558^{*}$ \\
\hline & & $\mathrm{p}$ & 0.005 & 0.002 \\
\hline & \multirow{2}{*}{$1: 0: 1$} & $\mathrm{r}$ & $0.573 *$ & $0.658^{*}$ \\
\hline & & $\mathrm{p}$ & 0.003 & $<0.001$ \\
\hline & \multirow{2}{*}{ EXP } & $\mathrm{r}$ & 0.314 & 0.358 \\
\hline & & $\mathrm{p}$ & 0.135 & 0.086 \\
\hline \multirow{6}{*}{ Trunk FFM } & \multirow{2}{*}{$2: 0: 2$} & $\mathrm{r}$ & -0.053 & -0.106 \\
\hline & & $\mathrm{p}$ & 0.850 & 0.622 \\
\hline & \multirow{2}{*}{$1: 0: 1$} & $\mathrm{r}$ & 0.114 & 0.211 \\
\hline & & $\mathrm{p}$ & 0.596 & 0.323 \\
\hline & \multirow{2}{*}{ EXP } & $\mathrm{r}$ & $0.441 *$ & $0.457^{*}$ \\
\hline & & $\mathrm{p}$ & 0.031 & 0.025 \\
\hline
\end{tabular}




\section{Discussion}

Push-up exercise is popular and widely usage type of exercise. In addition, it is possible to achieve more positive gains with different variations. It consists of two main phases and many muscles work as active primary mover, synergists. One of the most important variables affecting the muscle activation and gain from the exercise is velocity/tempo of movement. In the studies carried out, different results were reported at different tempo and these differences were revealed $[5,15]$. However, no cumulative assessment was presented for the dominant and nondominant side in terms of range of motion (ROM), vertical ground reaction force (vGRF), joint velocity and displacement. For this reason, the most frequently using tempos in resistance exercise programs such as 1:0:1, 2:0:2, explosive tempo (EXP) were preferred, and they compared to each other. As a result, it was aimed to reveal data that would help practitioners while performing the push-up in their exercise programs.

The results showed that the dominant shoulder displacement was found minimum in EXP. However, the shoulder displacement in the other two tempos was similar. There was an average of $9 \%$ variation between the explosive tempo and the other two tempos. While 1:0:1 and 2:0:2 were fixed tempos, the eccentric phase of EXP was average $0.37 \pm 0.07 \mathrm{~s}$, the concentric phase was $0.45 \pm 0.1 \mathrm{~s}$ and the push-up was completed in $0.82 \pm 0.15 \mathrm{~s}$. Although the 1:0:1 tempo was completed in half time of the 2:0:2, the shoulder displacement was almost the same. Although these two tempos are very different from each other, their effects on shoulder displacement were not observed. The highlight here is that EXP reduces shoulder displacement compared to the other two tempos and makes a difference considering the 1:0:1 tempo. When the elbow displacement was examined, the results in the shoulder displacement was similar. In elbow displacement, the lowest value belongs to EXP and there was $9 \%$ variation difference compared to the other two tempos. Among the reasons why the EXP had the lowest shoulder and elbow displacement, it may be considered that the eccentric and concentric phases of the movement were performed at the maximal speed without the metronome.

Another variable that supports this situation was the results obtained from the right and left ROMs. Similar to the displacements of the joints, the lowest values in ROMs were also observed in EXP. EXP made difference for ROMs in comparison with the tempo of 1:0:1 and 2:0:2. The lowest values of EXP tempo in displacements and ROMs may be thought of as a result of trying to perform the movement at maximum speed and that the participant did not fully complete the eccentric and concentric phases as in the tempo of 1:0:1 and 2:0:2. This might cause less muscle activation in EXP, and the fatigue occurs later in push-up due to the inability to use full ROM. Hsu et al. [8] observed that fatigue occurs later at fast tempos. This situation may be supported by this study with less used ROM and joint displacement. In some studies, it has been showed that there is more muscle activation in slower tempos $[1,7,15]$. It has been found that exercise performed at slower tempos decreases the load on the elbow joint and increases muscle activation [1]. It may be said that EXP creates a more disadvantageous situation in terms of muscle activation and hypertrophy. When the loads on the elbow joint were examined, it was found that the tempo applied faster during the exercise puts more load on joints [1]. But in this case, it should not be ignored that it is still a popular tempo for developing upper-body power in this tempo [16]. However, according to this study, it is clearly observed that attention should be paid to avoid undesirable consequences during push-up in EXP.

The velocity differences between the descent and ascent phases in the shoulder and elbow joints were examined, it was observed that the velocity difference increased with the increase in tempo. In the shoulder joint, there was 393\% difference between EXP and 2:0:2. In the elbow joint, this difference was found to be approximately $230 \%$. This may indicate that the movement is applied with more irregular kinematics as the difference between the descent and ascent phases increases in EXP. However, the small difference between the descent and ascent phases at 2:0:2 indicates that the movement is more controlled at this tempo. When vGRFs were also examined, it was observed that the increase in tempo increased the peak vGRFs. Significant differences were found between each tempo in peak vGRFs. This shows that the effect of tempo changing on vGRF. When the effect of tempo on ascent-descent velocity differences, vGRF, ROM and joint displacements was evaluated, it was observed that tempo had greater effect on vGRF. When the mean vGRF data were examined, there was a 6\% significant difference between 1:0:1 and EXP only in the right-side descent phase. However, in the average vGRF occurring at 2:0:2 and 1:0:1 tempo, it was observed that the averages of the descending phases were approximately 3.7\% higher than the ascending phases. On the other hand, the ascent phases in EXP were found to be approximately $1.5 \%$ more than the descent phases. This may indicate that in EXP, the participants released themselves with gravity during the descent phase and weigh upon the load cells more quickly and effectively in the ascent phase, so that the exercise could be performed quickly.

At the same time, muscle mass has been found to have an effect on peak vGRFs. Significant positive correlations were observed between right and left arm lean muscle mass and peak vGRF values in 1:0:1 and 2:0:2. In this case, the higher muscle ratio may indicate that there was no provide increase in peak vGRFs in the push-up exercise 
performed at a faster tempo. In other words, the muscle ratio of the individuals does not matter in terms of vGRF in EXP. In EXP, vGRF was high; it seems more likely to be explained by effects such as motion velocity, gravity, inertia. However, in EXP; an interesting result was also observed regarding the right and left arm total FFM, trunk FFM and observing the percentage of peak vGRF repetition to total repetition. The increase in trunk and arm FFM caused peak vGRF to be observed in further repetitions. When these situations are evaluated together, it may be said that FFM has no effect on the high peak vGRF value in EXP, but it has an effect on obtaining the peak value in further repetitions. In a study, found that fatigue occurs later at a faster tempo [8]. In this case, it may be said that there may be a relationship with the onset of fatigue in obtaining the peak vGRF values in further repetitions.

\section{Conclusion}

According to this study, 2:0:2 tempo may be one of the safest and most beneficial for practitioners. In addition to this, as the tempo increases, the joint displacement and ROM decrease, but the peak vGRF increase. Tempo of the movement should be slow to minimize the difference between joint velocity in the descent and ascent phases and to perform the movement using more range of motion. In addition to this situation, the peak vGRF values of individuals with more muscle mass were obtained in further repetitions. Therefore, practitioners should perform the exercise more carefully and determine the training goal to avoid undesirable results in 1:0:1 and higher tempos.

Conflict of interest: Authors state no conflict of interest.

\section{References}

1. Chou P.P.-H., Hsu H.-H., Chen S.-K., Yang S.-K., Kuo C.-M., Chou Y.-L. (2011) Effect of push-up speed on elbow joint loading. J. Med. Biol. Eng., 31(3): 161-168.

2. Cogley R.M., Archambault T.A., Fibeger J.F., Koverman M.M. (2005) Comparison of muscle activation using various hand positions during the push-up exercise. J. Strength Cond. Res., 19(3): 628.

3. Cohen J. (2013) Statistical power analysis for the behavioral sciences: Academic press.

4. Contreras B., Schoenfeld B., Mike J., Tiryaki-Sonmez G., Cronin J., Vaino E. (2012) The biomechanics of the push-up: Implications for resistance training programs. Strength Cond. J., 34(5): 41-46.

5. Dhahbi W., Chaabene H., Chaouachi A., Padulo J., Behm D.G., Cochrane J., Burnett A., Chamari K. (2018) Kinetic analysis of push-up exercises: a systematic review with practical recommendations. Sports Biomech., 21(1): 1-40.

6. García-Massó X., Colado J.C., González L.M., Salva P., Alves J., Tella V., Triplett N.T. (2011) Myoelectric activation and kinetics of different plyometric push-up exercises. J. Strength Cond. Res., 25(7): 2040-2047.

7. Gouvali M.K., Boudolos K. (2005) Dynamic and electromyographical analysis in variants of push-up exercise. J. Strength Cond. Res., 19(1): 146-151.

8. Hsu H.-H., Chou Y.-L., Huang Y.-P., Huang M.-J., Lou S.-Z., Chou P.P.-H. (2011). Effect of push-up speed on upper extremity training until fatigue. J. Med. Biol. Eng, 31: 289-293.

9. Kikuchi N., Nakazato K. (2017) Low-load bench press and push-up induce similar muscle hypertrophy and strength gain. J. Exerc. Sci. Fit., 15(1): 37-42.

10. Kowalski K.L., Connelly D.M., Jakobi J.M., Sadi J. (2021) Shoulder electromyography activity during pushup variations: a scoping review. Shoulder \& Elbow, 17585732211019373.

11. Lehman G.J., MacMillan B., MacIntyre I., Chivers M., Fluter M. (2006) Shoulder muscle EMG activity during push up variations on and off a Swiss ball. DM, 5(1): 1-7.

12. Maxwell S.E., Delaney H.D., Kelley K. (2017) Designing experiments and analyzing data: A model comparison perspective: Routledge.

13. Wang R., Hoffman J.R., Sadres E., Bartolomei S., Muddle T.W., Fukuda D.H., Stout J.R. (2017) Evaluating upper-body strength and power from a single test: The ballistic push-up. J. Strength Cond. Res., 31(5): 1338-1345.

14. Yang J., Christophi C.A., Farioli A., Baur D.M., Moffatt S., Zollinger T.W., Kales S.N. (2019) Association between push-up exercise capacity and future cardiovascular events among active adult men. JAMA Netw., 2(2): e188341-e188341.

15. Yoo W. (2014) Effect of exercise speed and isokinetic feedback on the middle and lower serratus anterior muscles during push-up exercises. J. Phys. Ther. Sci., 26(5): 645-646.

16. Zalleg D., Dhahbi A.B., Dhahbi W., Sellami M., Padulo J., Souaifi M., Beslija T., Chamari K. (2020) Explosive push-ups: from popular simple exercises to valid tests for upper-body power. J. Strength Cond. Res., 34(10): 2877-2885.

\section{Received 23.09.2021 \\ Accepted 30.11.2021}

(c) University of Physical Education, Warsaw, Poland

\section{Acknowledgments}

Authors would like to thank the participants. This research received no external funding. 